Kimon Howland Sargeant, Seeker Churches. Promoting Traditional Religion in a Nontraditional Way New Brunswick (NJ), Rutgers University Press, 2000, 189 p. (bibliogr., annexes, index).

Danièle Hervieu-Léger

\title{
CpenEdition
}

Journals

Édition électronique

URL : http://journals.openedition.org/assr/1314

DOI : $10.4000 /$ assr. 1314

ISSN : $1777-5825$

Éditeur

Éditions de l'EHESS

Édition imprimée

Date de publication : 1 avril 2003

Pagination : $59-157$

ISBN : 2-222-96732-5

ISSN : 0335-5985

Référence électronique

Danièle Hervieu-Léger, « Kimon Howland Sargeant, Seeker Churches. Promoting Traditional Religion in a Nontraditional Way ", Archives de sciences sociales des religions [En ligne], 122 | avril - juin 2003, document 122.46, mis en ligne le 10 novembre 2005, consulté le 24 septembre 2020. URL : http:// journals.openedition.org/assr/1314; DOI : https://doi.org/10.4000/assr.1314 
des endroits divers de l'ouvrage. Elle ne fait donc pas l'objet d'un chapitre ou d'une rubrique spéciale. C'est la question des « mœurs ». D'emblée, l'introduction insiste sur la nouvelle prise en compte par les musulmans de " valeurs » plutôt que de normes juridiques religieuses. Or les exemples cités relèvent du discours éthique : questions de l'avortement et de 1'homosexualité. "Ce qui était interdit mais vécu dans le non-dit devient soudainement un enjeu de société ». Les sociétés occidentales, vues par les islamistes radicaux, sont « corrompues » (p. 25). «La réislamisation des sociétés se fait sous des formes conservatrices (portant sur le droit et les mœurs) : elle a permis l'émergence de nouveaux acteurs qui ne s'inscrivent pas dans la perspective d'une gestion du pouvoir étatique » (p. 30) «En fait l'islamisme au pouvoir devient conservateur et rejoint le néofondamentalisme sur un point : la question des mœurs devient centrale.» (p. 40) «Le néofondamentalisme est parfaitement compatible avec la globalisation, dans sa forme libérale cette fois-ci, ne posant comme limite que la question des mœurs et de la morale, thème que l'on retrouve aussi dans la droite religieuse américaine ». Même si l'A. dénie au phénomène une spécificité absolue, il note, parmi les musulmans européens « une valorisation de l'honneur et de la pudeur des femmes, une réticence à la mixité » (p. 60). On pourrait continuer en citant les passages consacrés à « l'islam humaniste " où transparaît l'insistance sur des valeurs de " morale pratique ", de "réalisation de soi » (question de la drogue, du tabac, du respect du corps, des qualités personnelles etc.). Cette question de l'ordre éthique dans la société, au niveau social et individuel, aurait mérité, nous semble-t-il, une analyse plus attentive et articulée avec les manifestations de l'islam politique. Parce que c'est sans doute à ce niveau que se trouvent l'enracinement le plus large et la base sociale la plus étendue pour tous les mouvements de réislamisation de ces dernières décennies. Le phénomène d'appel à la restauration des mœurs (al-akhlâq) apparaît comme un des plus récurrents de l'histoire des mouvements islamiques, se déclenchant soit aux moments de l'islamisation ou des colonisations (les païens ou les Européens ont des mœurs corrompues) soit au moment des révolutions internes (le pouvoir n'est plus musulman). Et dans la plupart des cas de figure où la documentation est explicite, les prêches et les actions islamiques contre les mauvaises mœurs ont été le marchepied d'une mobilisation générale et d'une prise du pouvoir violente (le paradigme est Ibn Tumart).

Sans qu'on puisse en faire le tour, cet ouvrage soulève des questions majeures à propos de l'évolution du système religieux islamique, particulièrement au niveau des mouvements de pensée et d'action les plus entreprenants. Sur le fond, peut-on dire que l'occidentalisation, la déculturation et l'individualisation sont partout aussi présentes que le suggère l'ouvrage? Les passages consacrés aux «néoconfréries » auraient permis s'ils avaient abordé plus largement le phénomène confrérique, de montrer qu'il existe de formidables poussées qui vont en sens inverse. Depuis les années 1970 , on ne peut que constater un profond mouvement de patrimonialisation locale et régionale des manifestations rituelles ou festives de l'islam, qui s'est produit, entre autres, par réaction identitaire face à une modernisation occidentalisante. Mais on ne peut que savoir gré à l'A. d'avoir, de façon aussi stimulante et novatrice, mis en lumière ces tendances de fond de l'islam politique contemporain.

Signe des temps, si la bibliographie (de bas de page) n'est pas très fournie, celle des sites web l'est beaucoup plus.

\section{Constant Hamès.} SARGEANT (Kimon Howland).

Seeker Churches. Promoting Traditional Religion in a Nontraditional Way. New Brunswick (NJ), Rutgers University Press, 2000, 189 p. (bibliogr., annexes, index).

Comment gérer le besoin de participation communautaire des "seekers", ces "chercheurs de sens" de plus en plus nombreux qui rejettent toute appartenance dénominationnelle, mais souhaitent pouvoir inscrire leur quête personnelle dans des formes d'expression collective renouvelées? Un certain nombre de nouvelles Églises évangéliques américaines se sont constituées, depuis une dizaine d'années, avec le projet précis d'attirer ces nouveaux consommateurs spirituels en leur offrant des produits culturels correspondant à une demande qui ne trouve plus sa satisfaction du côté de l'offre religieuse traditionnelle. L'étude de K.H.S. s'attache au cas de la Willow Creek Association, créé à cette fin par la Willow Creek Community Church près de Chicago et qui a connu en huit ans un spectaculaire développement en devenant - avec plus de 5000 implantations - la référence du "seeker church movement". Si l'image du "super marché spirituel" est couramment utilisée pour décrire le paysage religieux contemporain, elle perd ici son caractère métaphorique. Elle correspond en effet à une réalité revendiquée comme telle par les promoteurs du projet : celui de constituer un "shopping mall center" dans lequel des individus, dont l'aspiration et la capacité consommati- 
ves sont fortes mais le choix peu fixé, peuvent trouver les produits spirituels les mieux ajustés à leurs attentes. Des produits dont la définition principale est de servir, de façon directe et immédiate, à l'accomplissement personnel des intéressés. Croire et "se sentir bien" - dans sa vie, dans son groupe, dans sa tête et dans son corps - tel est le mot d'ordre de ce management ultra-moderne des biens spirituels, qui embrasse à la fois des propositions cultuelles (des liturgies utilisant toutes les ressources de la technologie la plus moderne du spectacle et de la communication), des propositions thérapeutiques, des offres de conseil touchant à tous les domaines de la vie quotidienne (de la vie relationnelle aux placements financiers) et des activités récréatives diverses.

L'intérêt du propos de H.K.S. ne réside pas seulement dans la description soignée des techniques pastorales à tout le moins peu classiques que mettent en œuvre les seekers churches construites sur le modèle de la Willow Creek Association. Car ces Églises gérées comme des entreprises de services flexibles, décentralisées et gouvernées par la recherche de l'innovation permanente, ne se contentent pas simplement de "rajeunir" une offre traditionnelle dans le domaine de la liturgie et de l'accompagnement personnalisé des fidèles, avec l'objectif de "fixer" la masse flottante des "chercheurs de sens" au sein d'une forme classique d'une communalisation religieuse de type Église. En s'ajustant d'aussi près que possible aux besoins ressentis et exprimés par les individus auxquels elles s'adressent, elles contribuent à faire émerger un nouveau type de croyants qui conduisent explicitement leur recherche spirituelle comme une démarche consumériste mettant en jeu des critères d'appréciation et des procédés de décision relevant entièrement d'une logique économique de marché. Elle suscite également un mode original de sociabilité religieuse dont le ressort est la consommation collective des biens offerts de façon personnalisée à chacun.

Cette modalité particulière d'engagement religieux individuel assumé en groupe entre en affinité avec des formes de participation associative profane qui correspondent à la culture $\mathrm{du}$ public des seekers. Elle favorise la stabilisation (relative) de communautés d'un genre nouveau, liées moins par leur adhésion à un corps de croyances partagées que par le choix d'un style de consommation commun : des coopératives de consommateurs spirituels, en quelque sorte, parfaitement adaptées, selon l'auteur, au mode de vie des classes moyennes urbaines dont sont issus ceux qui les fréquentent. Le propre de ces Églises est donc d'assurer une parfaite continuité entre la vie profane - orga- nisée par et autour de l'activité consommative et une vie religieuse qui, loin d'éloigner les fidèles du monde, en épouse au contraire toutes les caractéristiques les plus contemporaines et s'emploie à fournir aux croyants une direction de vie pragmatique leur assurant, autant que possible dès maintenant, bonheur, harmonie familiale et estime de soi. Ces coopératives de consommation spirituelle (c'est nous qui les nommons ainsi) conservent-elles un quelconque apparentement avec les Églises évangéliques dont elles sont issues? Maintiennentelles (et encouragent-elles) un enracinement effectif dans une mémoire chrétienne? L'A. montre bien qu'il existe une tension entre le souci d'une adaptation réactive aux attentes contemporaines mise en œuvre au sein du seekers church movement et la préservation d'un ancrage dans une tradition religieuse historique, inscrite dans des structures dénominationnelles. La conservation assez généralement observée dans ces groupes d'un corps minimum de références théologiques et morales (au demeurant plutôt conservatrices) n'est pas incompatible avec une dévaluation de la tradition historique dont ces références et valeurs sont issues.

Le succès effectivement remarquable des seekers churches semble bien procéder de la combinaison qu'elles réalisent entre une indiscutable inventivité expressive (en matière de musique, dramaturgies, spectacles interactifs, etc.) et un conformisme religieux et moral efficacement sécurisant : une combinaison parfaitement ajustée à des dispositions et attentes sociales petites-bourgeoises recyclées dans la culture ultra-moderne du soi. L'A., qui fournit tous les éléments d'un tel diagnostic sans le formuler vraiment, est manifestement trop fasciné par le succès d'audience de ces groupes pour en explorer les implications. Qu'il s'agisse d'un phénomène de masse ne fait aucun doute. Que ce phénomène - qui se présente comme une formidable boîte à écho de l'univers contemporain de la consommation - soit porteur, comme tel, "d'un impact culturel majeur" reste à démontrer. L'idée qu'il pourrait s'agir d'un "Réveil moderne", voire même comme d'une "seconde Réforme", qui serait basée "non sur la doctrine, mais sur la forme" laisse quelque peu rêveur, s'agissant précisément d'un mouvement immergé dans la culture du présent au point d'apparaître dépourvu, non seulement de toute portée protestataire, mais même de cette distance culturelle minimale, indispensable à l'émergence d'une quelconque créativité sociale. On demeure tout aussi sceptique lorsque l'A. suggère, dans les dernières pages de son ouvrage, que les seeker churches, pleinement héritières de "la stratégie évangélique qui consiste à changer le monde en changeant les 
individus", pourraient bien constituer un phare du protestantisme évangélique américain, susceptible de relayer dès maintenant l'effet Billy Graham. Si ces considérations généralisantes semblent un peu douteuses, on admettra cependant volontiers, en suivant l'A., que les seekers churches constituent un terrain fascinant pour l'analyse des formes ultra-modernes de la communalisation spirituelle, et un contrepoint nécessaire aux études portant sur les logiques intégralistes et antimodernes à l'œuvre dans les courants fondamentalistes de la nébuleuse évangélique américaine.

Danièle Hervieu-Léger.

\subsection{7}

SCHMITT (Jean-Claude).

Le Corps des images. Essais sur la culture visuelle au Moyen Âge. Paris, Gallimard, 2002, 409 p. (illustr.) (coll. « Le temps des images $»)$.

J.-C.S. a réuni dans ce livre onze études, pour la plupart inédites en français. Chacune étayée, selon la norme de la collection, sur un riche dossier iconographique, elles reflètent quinze ans de ses recherches sur le statut de l'image dans le christianisme latin au Moyen Âge. L'importance de cette question pour la compréhension du religieux chrétien n'est plus à démontrer : loin de concerner le seul domaine de l'histoire de l'art, elle donne accès à des débats théologiques aussi centraux que ceux concernant la ressemblance entre l'homme et Dieu, l'Incarnation ou encore, à travers les théories médiévales du rêve et des visions, les expériences sensibles du surnaturel. Jusqu'à ces dernières années, l'importance des enjeux de l'image avait surtout été mise en lumière à propos du christianisme byzantin, avec la crise iconoclaste du $\mathrm{VIII}^{\mathrm{e}}$ siècle débouchant sur le Concile de Nicée II en 787 et l'élaboration d'une théologie de l'icône, qui explicite sa valeur religieuse et définit les contours d'un véritable «art sacré». En éclairant le cheminement des images dans le christianisme latin, les travaux de J.-C.S. apportent depuis plusieurs années déjà une contribution essentielle à ce domaine d'études jusqu'ici réservé, pour l'essentiel, aux historiens de l'art. Ils autorisent aussi des ouvertures comparatives très riches, comme en témoigne dans cet ouvrage l'étude "Liberté et normes des images occidentales », qui montre en particulier comment un encadrement théologique moins rigoureux a contribué largement à ouvrir, en Occident, la carrière d'un art chrétien inventif et bientôt accessible, faute d'une sacralisation explicite des images, à un processus d'" émancipation », pour reprendre un terme de l'auteur, lui-même préalable à l'émergence d'un registre sécularisé de l'art.

Le déficit de légitimité théologique dont ont pâti les images dans le christianisme latin ne veut pas dire qu'elles soient dépourvues de toute sacralité, bien au contraire. Mais, au lieu d'adopter la voie d'une théologie complexe et valorisée de l'image, leur légitimité semble s'être construite, dans une large mesure, à partir des pratiques dévotionnelles effectives et dans le registre discursif de la légende. Décisifs, à ce double point de vue, sont les écrits et la légende de Grégoire le Grand (590-604), analysés dans le chapitre "Écriture et image ». "La lettre écrite en l'an 600 par le pape Grégoire à l'évêque iconoclaste Serenus de Marseille peut être considérée comme le fondement de l'attitude de l'Église d'Occident à l'égard des images religieuses ", écrit J.-C.S. (p. 101). C'est à lui que l'on doit la métaphore de la «lecture»" des images par les ignorants, qui les fera désigner plus tard comme la «Bible des illettrés ". Grégoire leur reconnaît aussi une valeur affective : la «componction» qu'elles suscitent favorise la prière et l'amour de Dieu.

Ces justifications restent minces, comparées à la théologie néo-platonicienne du transitus, l'idée que l'on peut atteindre les réalités invisibles au moyen des choses sensibles. Mais elles suffisent précocement à faire de Grégoire l'autorité en matière d'images puisque c'est une autre de ses lettres qui, au VIII ${ }^{\mathrm{e}}$ siècle, est enrichie d'une interpolation d'esprit néoplatonicien et est, sous cette forme, impliquée désormais dans les débats sur la légitimité du culte des images. La date de la falsification est significative. Comme le rappelle un chapitre précédent, en effet, à propos des répercussions en Occident du second Concile de Nicée, le culte des images ne va pas de soi pour une religion qui, en principe, reconnaît sans réserve le Décalogue et donc ce commandement divin : «Tu ne feras point d'images taillées (...) et tu ne te prosterneras pas devant elles » (Exode, XX, 3-4). Des tendances iconoclastes, anciennement attestées, n'en finiront pas de renaître dans le christianisme (jusqu' aux suites de Vatican II !). Elles sont sensibles, au VIII ${ }^{\mathrm{e}}$ siècle, dans les Libri Carolini, très critiques à l'égard de l'icônodulie des Orientaux telle que la justifie Nicée II. On comprend donc que, dans les controverses suscitées par ces textes, l'autorité de Grégoire ait été invoquée par les partisans des images, même s'il avait fallu pour cela modifier sensiblement son point de vue!

De fait, et c'est là une des thèses centrales de l'A., le triomphe des images s'enracine dans l'évolution des pratiques dévotionnelles, où elles jouent un rôle toujours croissant. Les 